\title{
Should hysterectomy be performed for the treatment of endometriosis?
}

\author{
Abri de Bruin \\ Department of Obstetrics and Gynaecology, University of Pretoria, Pretoria, South Africa
}

When there is a medical or surgical indication for a

hysterectomy apart from endometriosis the following discussion is not really relevant. The question deals with the role of hysterectomy purely for the management of endometriosis. Women, who have pelvic pain due to endometriosis that is unresponsive to conventional therapy that can include surgery or medication, are often advised to have a hysterectomy with or without a BSO. Age, gravidity and parity must be carefully considered when deciding on a hysterectomy and especially more so when a decision has to be taken surrounding a BSO.

A study done in 1999 had the following conclusions on this topic:

"Women who undergo hysterectomy for pelvic pain and endometriosis at $<30$ years old are more likely than older women to have residual symptoms, to report a sense of loss, and to report more disruption from pain in different aspects of their lives".'

\section{What is the definition of endometriosis?}

Endometriosis is the "occurrence of endometrial stroma and glands outside the uterus". ${ }^{2}$ That puts the disease location outside the uterus! So if the disease per definition is outside the uterus, then why would we want to remove the uterus?

\section{What is the cause of endometriosis?}

Despite numerous research papers, we still lack a definite answer. There are some theories and essentially, it comes back to retrograde menstruation and metaplasia.

If metaplasia is the main cause, then a hysterectomy will achieve nothing. If retrograde menstruation is the main cause, then stopping the retrograde menstruation by doing a tubal ligation, or medical induction of amenorrhea will be sufficient and a patient will not need a hysterectomy.

\section{What types of endometriosis can benefit from a hysterectomy?}

Peritoneal endometriosis will not be treated by removing a separate organ, the uterus. The same is true for ovarian endometriosis. Here it is crucial to remove the endometrioma to protect the ovarian function. Recto-vaginal endometriosis will

\section{Correspondence: \\ Dr AK de Bruin \\ email: drabri@webmail.co.za}

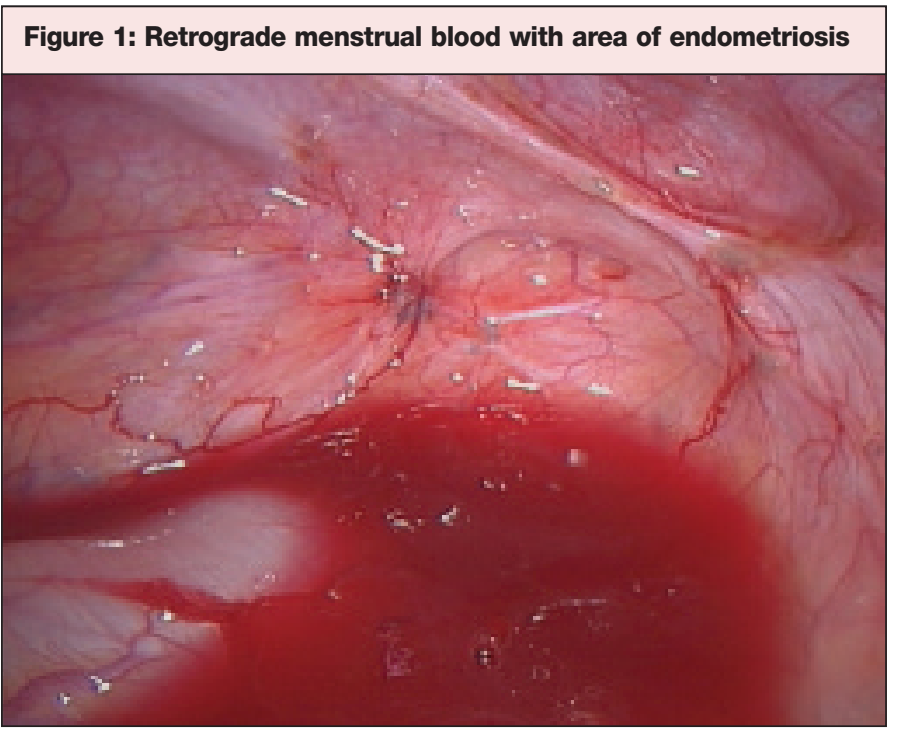

also not benefit. There is an argument to be made for the place of a hysterectomy following complete removal of all the endometriosis in a patient with recto-vaginal endometriosis.

In essence, not one of the forms of endometriosis will benefit from removing the uterus alone without treating the existing endometriosis.

\section{Does a uterus cause dyspareunia?}

In some instances this can be so. This is more so in a patient with a retroverted or retroflexed uterus. Should one then consider rather doing a ventro-suspension than a hysterectomy? The pros and cons of both procedures as well as outcomes and implications and complications should be discussed with the patient and the decision should be based on informed consent.

\section{What does a hysterectomy achieve?}

Amenorrhea is the main effect after hysterectomy.

Decrease in pelvic pain / discomfort if the uterus was the cause of the pain / discomfort

Loss of an organ.

\section{Is the amenorrhea of a hysterectomy preferable to} medical treatment?

This depends on the patient's history and wishes. Using GnRHa, the same can be achieved without reverting to a surgical procedure. 


\section{Can a Mirena ${ }^{\circledR}$ be used to cause amenorrhea?}

Yes it can as it will in most cases lead to decreased vaginal bleeding or amenorrhea and improved symptoms. One small study has shown that postoperative use of the LNG-IUS reduces the recurrence of painful periods in women who have had surgery for endometriosis. There is a need for further welldesigned RCTs of this approach.

\section{Should a BSO be done at the time of the hysterectomy?}

If we agree that endometriosis is to a certain extent dependant on estrogen stimulation, then a bilateral oophorectomy will remove the estrogen supply. However, the patient will have all the negative effects of being 'menopausal' and the risks associated with that. The end result will be that the patient will need to be put on estrogen replacement to improve her quality of life and then we will again be supplying estrogen to fuel the endometriosis. Remember that this is a disease of young women in their reproductive years therefore this treatment is definitely not ideal and should be considered if all else fails and the patient has been fully informed about all the risks and possible negative effects that she may experience.

According to the Cochrane Database "Hormone replacement therapy for women with endometriosis in post-surgical menopause could result in pain and disease recurrence. However, the evidence in the literature is not strong enough to suggest depriving severely symptomatic patients from this treatment. There is a need for more randomised controlled studies."

Endometriosis can present with a variety of symptoms including pelvic pain, dyspareunia and pain with defecation, up to several years after hysterectomy and bilateral salpingooophorectomy. This may occur when all endometriotic tissue is not excised at the time of the initial procedure. As excision of endometriosis at this time would be preferable, we have found laparoscopic excision of residual endometriosis to be effective in relieving endometriosis associated pain. ${ }^{3}$

Female castration without removing the surrounding fibrotic tissue can result, even if infrequently, in persistence of symptoms. ${ }^{4}$

\section{If one of the arguments for a hysterectomy is the fact that recto-vaginal endometriosis grows from the retro- cervical area - then}

At the time of surgery one needs to remove the cervix - so a subtotal hysterectomy will not help. The disease also often involve the uterosacral ligaments and therefore a subtotal hysterectomy is not indicated. If there is no recto-vaginal endometriosis present then the hysterectomy is prophylactic and only helpful if one believes that retrograde menstruation causes endometriosis.

\section{What is the optimal treatment of endometriosis?}

It depends on the symptoms. Does the patient have fertility issues and accidental endometriosis is diagnosed, or does she have infertility and pain and endometriosis? The basis for treatment will be surgical management (complete ablation or excision) of the endometriosis.

The combined surgical approach of laparoscopic laser ablation, adhesiolysis and uterine nerve ablation is likely to be a beneficial treatment for pelvic pain associated with minimal, mild and moderate endometriosis. As only one trial is included in the analysis, this conclusion should be interpreted with caution. ${ }^{5}$
GnRH-a have a role to play in patients with severe endometriosis, where surgery is planned and the patient will go into an assisted reproduction treatment cycle following the surgery. The GnRH-a should be administered following the surgery and the patient stays on the treatment till the IVF/ICSI cycle

\section{Can a hysterectomy help for the pelvic pain?}

According to several authors, substantial pain relief can be achieved in patients where a hysterectomy is performed for chronic pelvic pain. ${ }^{6,7}$ So hysterectomy can help for pain, but it will not remove the disease located outside the uterus.

\section{Conclusions}

Endometriosis is a complex disease with a multitude of sequelae of which pain and sub-fertility are the two main issues. However, it also interferes with the patient's quality of life, not only in the very severe cases. Severe endometriosis should be referred to a center that has an interest in this type of disease as well as a multidisciplinary team to deal with the disease optimally. But not even in such a center will all patients have complete removal of all of the disease all of the time. Some patients just have such severe disease that complete surgery is not always possible and even dangerous.

Complete surgery, especially patients with recto-vaginal disease following a previous hysterectomy is difficult and dangerous due to the fact that the uterus was removed and the anatomy is disturbed. So ideally, we should aim to remove the disease prior to a hysterectomy.

Offering a patient a hysterectomy as the magic treatment and subsequent stopping of the disease is the wrong advice. We should aim at treating the patient optimally and to remove the disease as completely as possible. If a hysterectomy is part of the "complete surgery", or in patients with chronic pelvic pain where all other methods of treatment has failed, then it has a role to play. ${ }^{7}$ However, we should move away from the belief that the only treatment for severe endometriosis is a TAH and BSO.

Despite all the advances and research, we still do not know all of the answers, but the right advice and optimal treatment for each individual is of utmost importance.

\section{References}

1. Long-term outcome of nonconservative surgery (hysterectomy) for endometriosis-associated pain in women <30 years old. American Journal of Obstetrics \& Gynecology. 180(6 Pt 1):1360-3, 1999 Jun.

2. Pelvic pain and dysmenorrhea, 415, In: Novak's Gynecology 12th edition. Jonathan S Berek.

3. Recurrent pain after hysterectomy and bilateral salpingooophorectomy for endometriosis: evaluation of laparoscopic excision of residual endometriosis. British Journal of Obstetrics \& Gynaecology. 106(7):740-4, 1999 Jul.

4. Redwine DB. Endometriosis persisting after castration: clinical characteristics and results of surgical management. Obstet Gynecol 1994; 83: $405-413$.

5. Cohrane Database.

6. Beard RW, Kennedy RG, Gangar KF et al. Bilateral oophorectomy and hysterectomy in the treatment of intractable pelvic pain associated with pelvic congestion. Br J Obsteet Gynaecol 1991; 98:988-992.

7. Hills SD, Marchblanks PA, Peterson HB. The effectiveness of hysterectomy for chronic pelvic pain. Obstet Gynecol 1995; 86: 941 945 


\section{Case illustrations}

\section{Patient with previous TAH for severe endometriosis}

Recto-vaginal nodule in patient with previous total abdominal hysterectomy

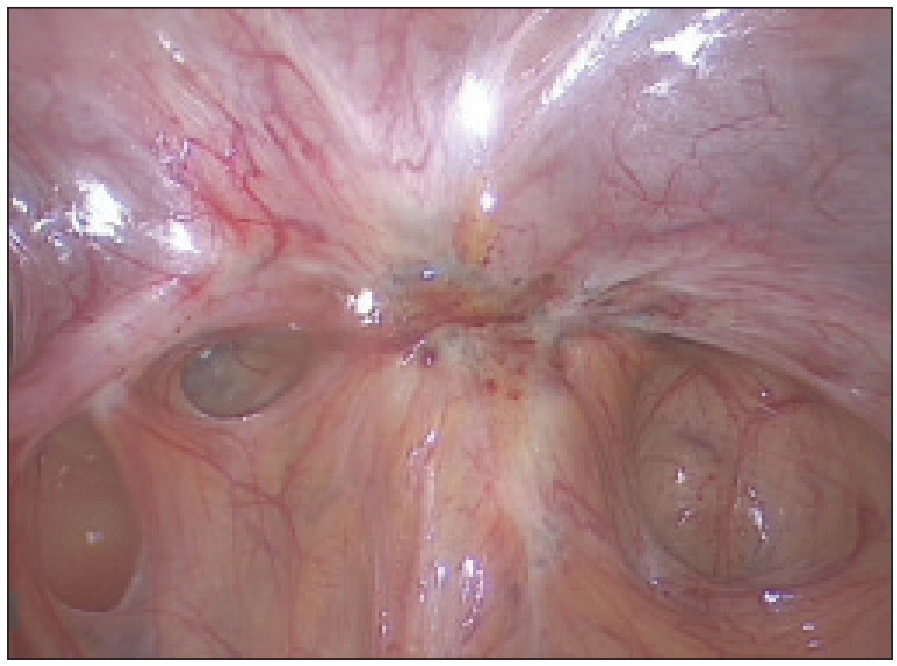

Opened recto-vaginal space

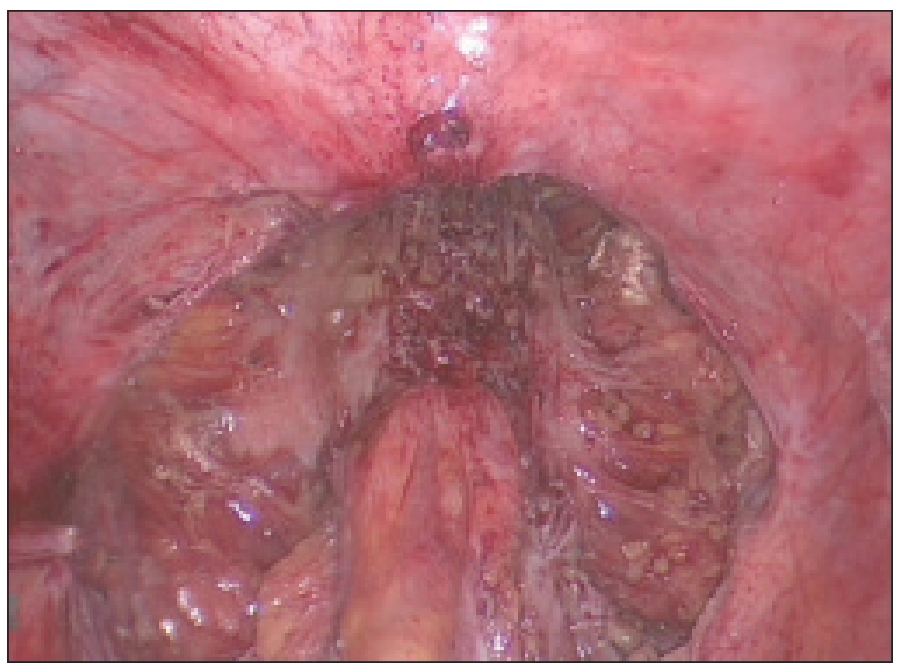

Infiltration of anterior rectal wall too low for resection. Superficial shaving was done



\section{Patient with recto-vaginal endometriosis and a normal} uterus

Normal uterus of patient with Recto-vaginal endometriosis

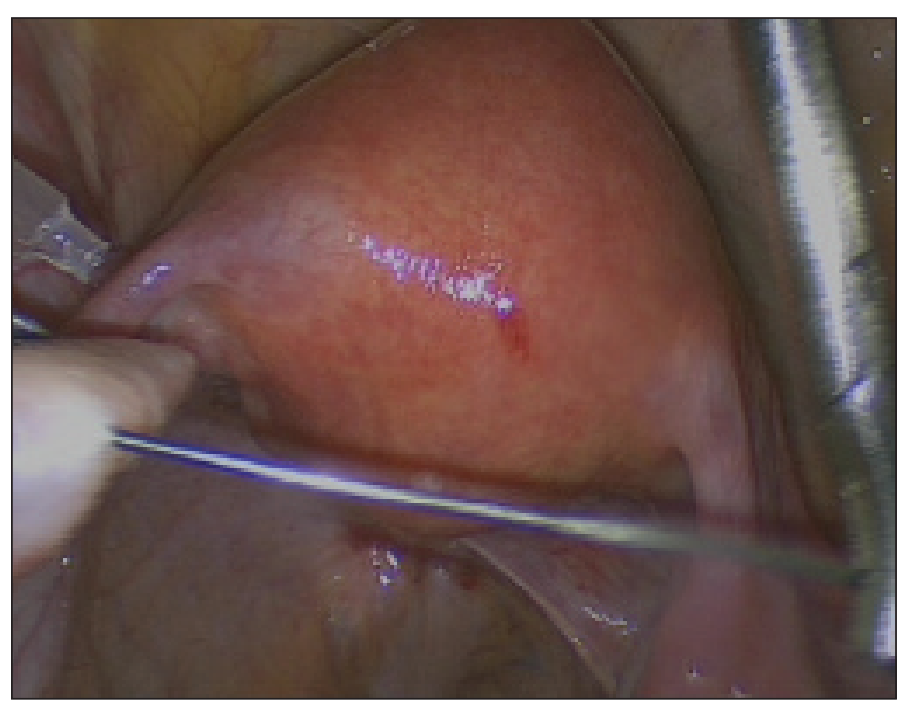

Rectum pulled up against back of vagina with anterior wall of Rectum infiltrated



Rectal wall shaved after mobilisation

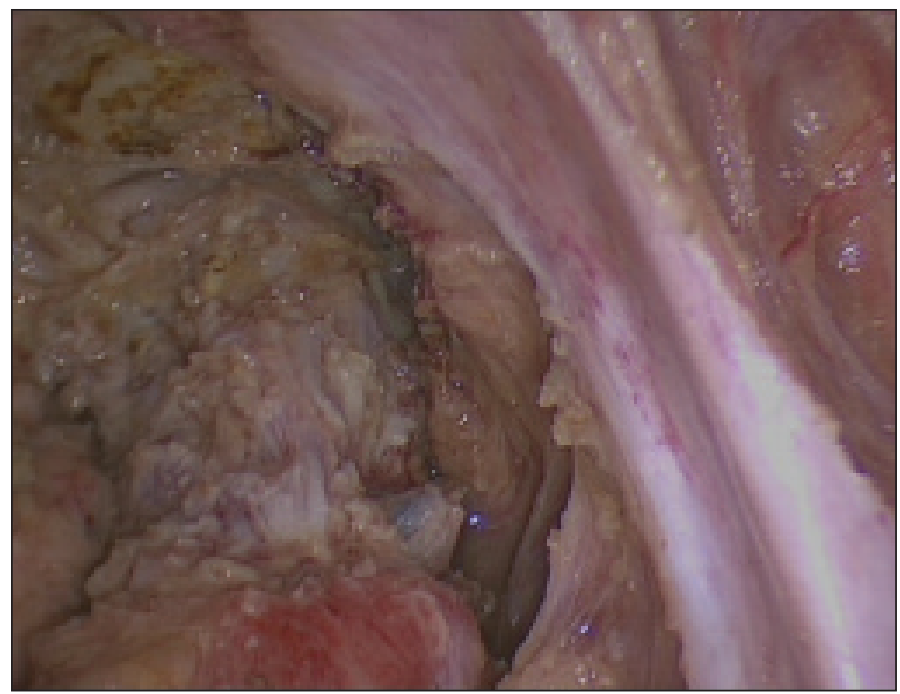




\section{Patient with previous vaginal hysterectomy for severe} endometriosis and now has a recto-vaginal nodule of endometriosis

Recto-vaginal nodule in patient following previous hysterectomy

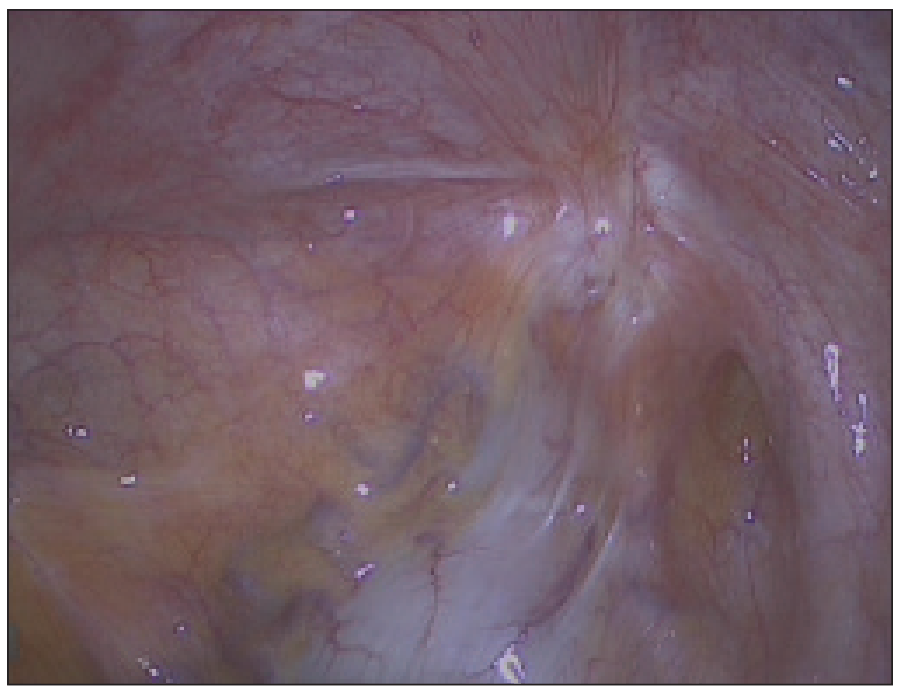

Infiltration of endometriosis in vaginal wall of same patient

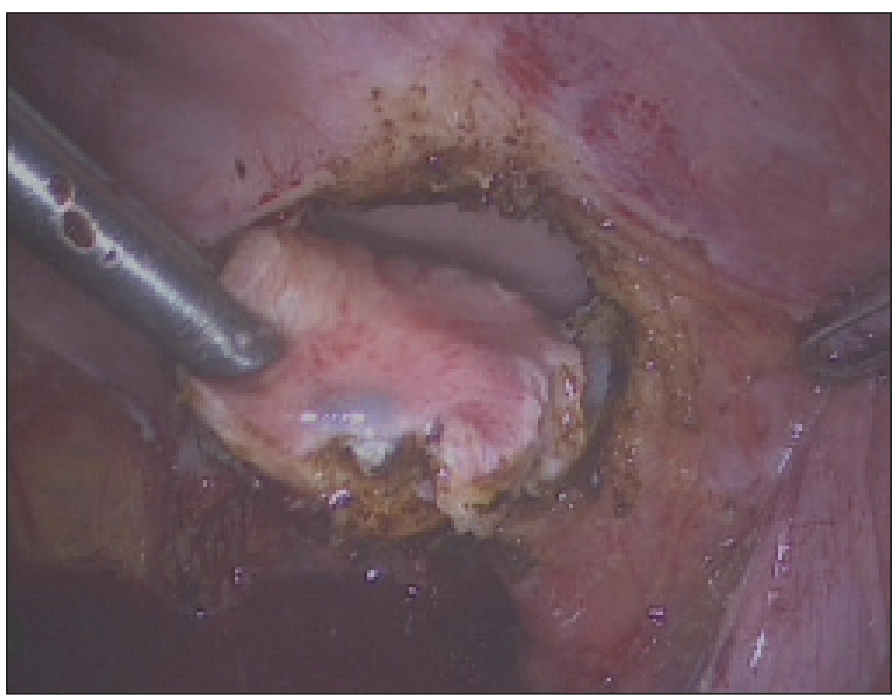

\section{Patient with previous subtotal laparoscopic} hysterectomy for severe endometriosis

RVE post laparoscopic subtotal Hysterectomy

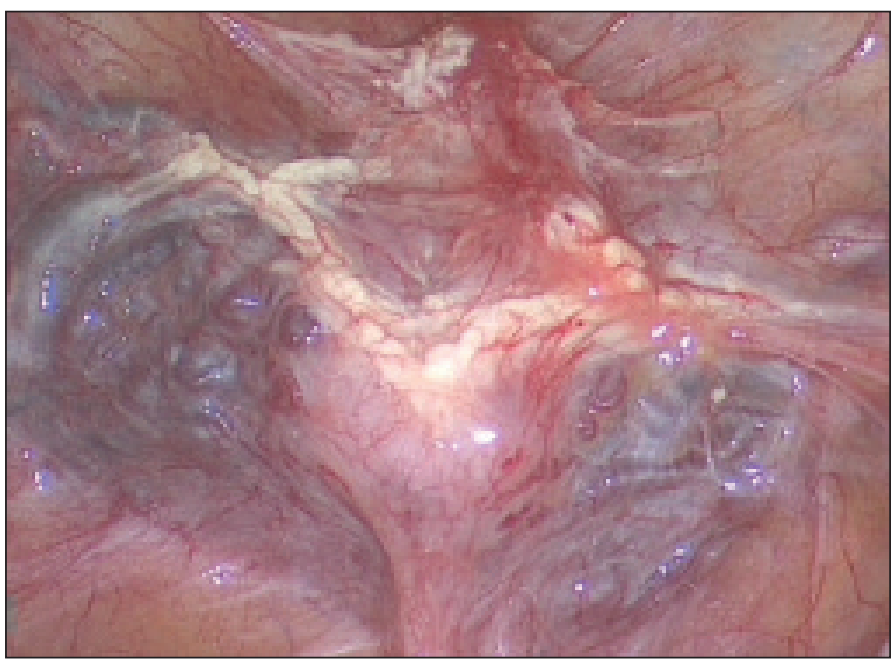

Rectum pulled up with no Recto-vaginal space visible

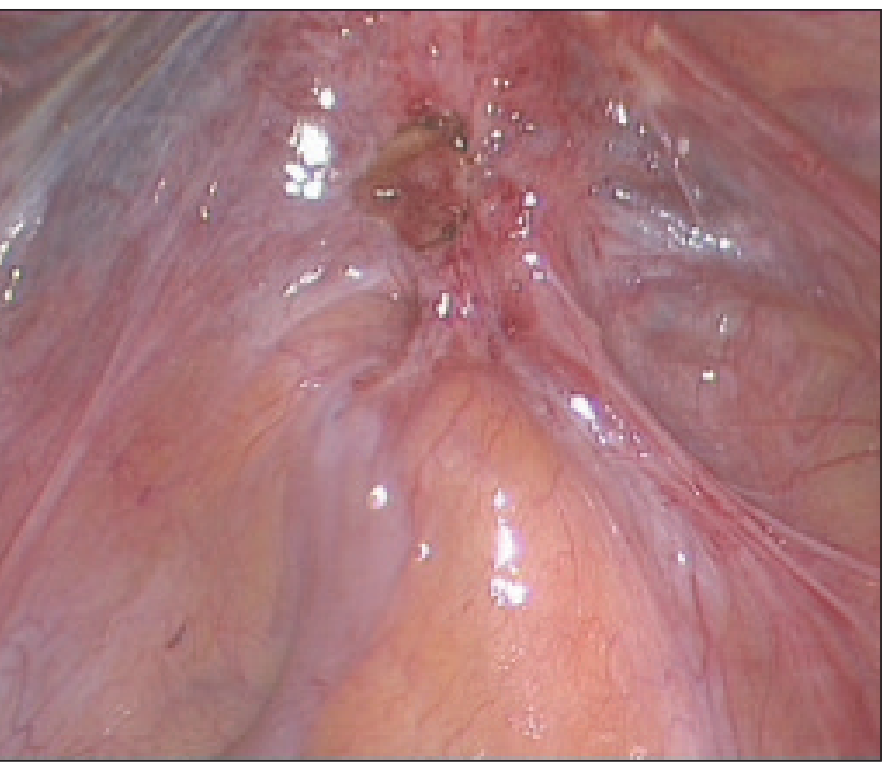

Restored recto-vaginal space

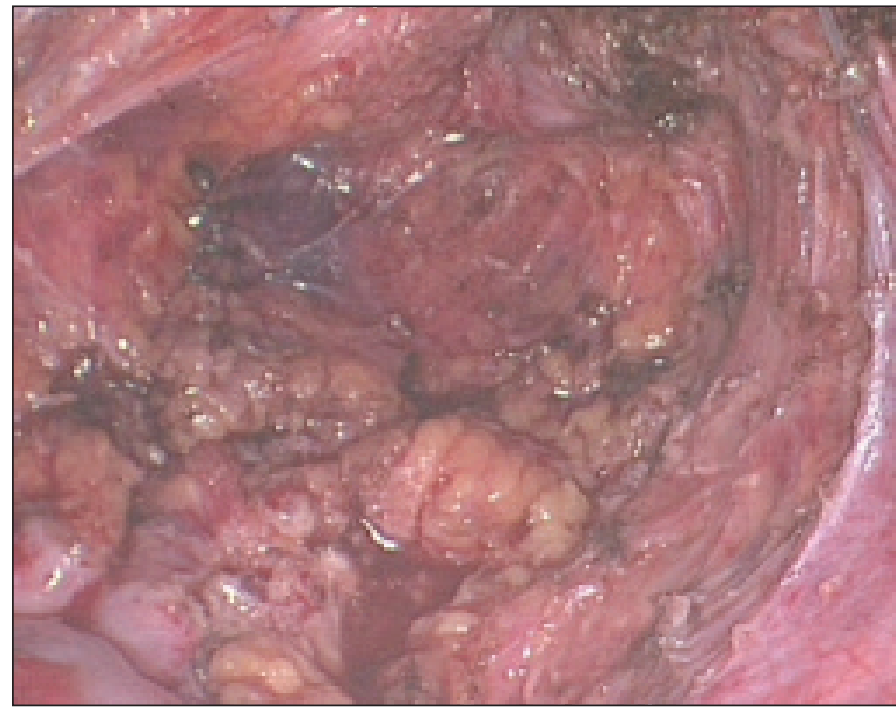

Area on side of rectum where nodule was resected

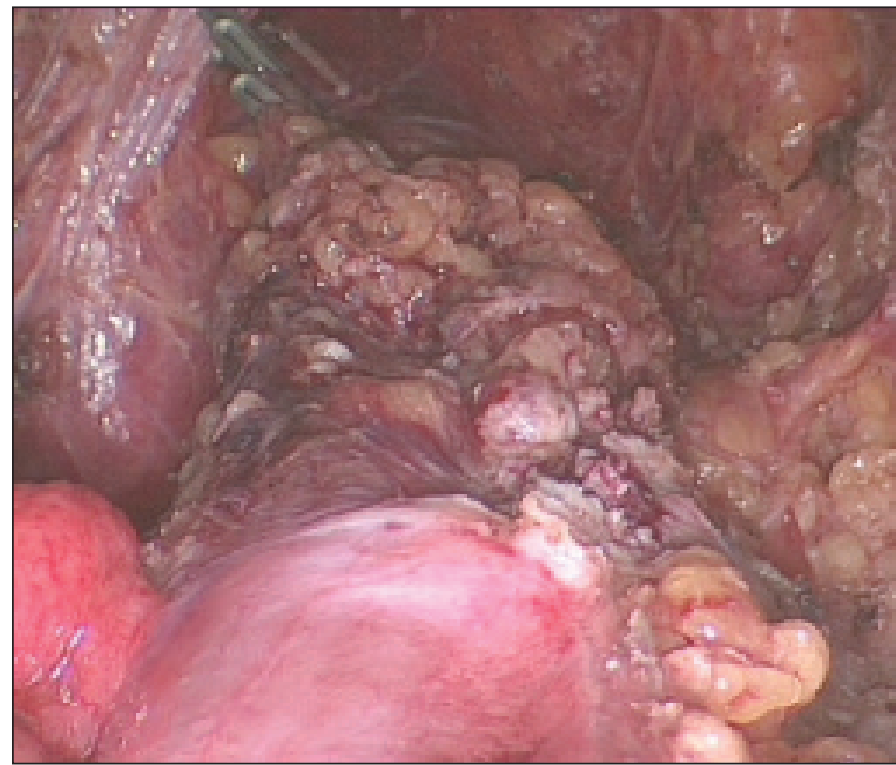

